\title{
Ventilatory Response to Exercise in Patients With Left-to-right Shunts
}

\author{
NIKOS GAZETOPOULOS* AND HYWEL DAVIES
}

\author{
From the Cardiac Department, Guy's Hospital, London S.E.1
}

An increased ventilatory drive is intimately related to dyspncea in most forms of heart disease (Campbell and Howell, 1963); knowledge of the mechanisms leading to an excessive ventilatory response is germane to the understanding of the pathogenesis of symptoms in such patients.

In previous communications we have discussed ventilation at rest and on exercise in relation to hæmodynamics and blood chemical changes, in normal subjects (Davies, Gazetopoulos, and Oliver, 1965), in several types of heart disease with closed cardiac septa (Gazetopoulos et al., 1966b; Gazetopoulos, Davies, and Deuchar, 1966a), and in cyanotic congenital heart disease (Davies and Gazetopoulos, 1965). Similar studies in patients with left-toright shunts remain to be described, the hæmodynamic changes with exercise having already been reported (Davies and Gazetopoulos, 1966). The relation between ventilation, arterial and venous blood chemistry, and hæmodynamics at rest and on effort, is discussed in this paper.

\section{SubJECTS AND METhodS}

The 34 patients included in this study had communications between the systemic and pulmonary circulations leading to the presence of a left-to-right shunt. The clinical data are presented in Table I. The techniques used for hæmodynamic measurement have been described in the previous paper (Davies and Gazetopoulos, 1966), and the account will, therefore, not be repeated. Ventilation was measured by means of a Wright respirometer at rest and during steady state after the fifth minute of exercise, the values being corrected to B.T.P.S. In parallel with the hæmodynamic and ventilatory measurements, arterial and venous blood samples were obtained in the third-to-fourth and eighthto-tenth minutes of effort. These blood samples were analysed for $p \mathrm{H}, \mathrm{Pco}_{2}$, and bicarbonate content.

Received July 21, 1965.

* Present address: Alex. Soutson 8, Athens 134, Greece.
Lactate and pyruvate determinations were made on arterial blood. These techniques have been previously described (Davies and Gazetopoulos, 1965).

In 10 patients we have studied the effects of heavier exercise on a bicycle ergometer in the sitting position. In 7 of these, rest and exercise data had also been obtained during cardiac catheterization in the supine position; in the other 3 , such data had been obtained only at rest. Exercise was begun at low exercise load (200 $\mathrm{kpm} . / \mathrm{min}$.) and was increased where possible by $200 \mathrm{kpm}$. $/ \mathrm{min}$. each 10 minutes, usually to the maximum exercise tolerance. Measurements of oxygen uptake, ventilation, heart rate, and arterial blood chemistry were made in similar sequence to that outlined above. The techniques employed for studies in the upright position have been the same as in normal subjects (Davies et al., 1965) and in patients with other forms of heart disease (Gazetopoulos et al., 1966a).

\section{RESULTS}

Mild Supine Exercise. Table I shows the ventilatory response at rest and on exercise in the supine position (hæmodynamic data have been given in the previous paper, the cases and their designations being the same). Table II shows the arterial and venous blood chemistry in those patients in whom measurements were made.

Ventilation and Hamodynamics. Figure 1 illustrates the ventilation in relation to the oxygen consumption, the types of lesion being designated by different symbols; the limits of normality are those taken from the published material and our data from this laboratory (Gazetopoulos et al., 1966b). Excessive ventilation is seen in a number of patients. Three showed slight arterial desaturation on effort due to an associated small right-to-left shunt: their ventilatory response did not appear to be unusual on this account.

In Fig. 2 we have plotted the deviation of ventilation from the mean normal for the given oxygen 
TABLE I

CLINICAL DATA, OXYGEN UPTAKE, VENTILATION, VENTILATORY EQUIVALENT, AND RESPIRATION RATE AT REST AND ON EXERCISE DURING CARDIAC CATHETERIZATION

\begin{tabular}{|c|c|c|c|c|c|c|c|c|c|c|c|c|c|c|}
\hline \multicolumn{3}{|c|}{ Case No., sex, and age } & $\begin{array}{l}\text { B.S.A. } \\
\left(\mathrm{m} .{ }^{2}\right)\end{array}$ & $\begin{array}{c}\mathrm{Hb} \\
\text { (g. } / 100 \mathrm{ml} .)\end{array}$ & Diagnosis & $\begin{array}{l}\text { Clinical } \\
\text { disability }\end{array}$ & \multicolumn{2}{|c|}{$\begin{array}{l}\text { Oxygen uptake } \\
\mathrm{ml} .^{2} / \mathrm{min} \text {.(STPD) }\end{array}$} & \multicolumn{2}{|c|}{$\begin{array}{c}\text { Ventilation } \\
1 . / \text { min.(BTPS) }\end{array}$} & \multicolumn{2}{|c|}{$\begin{array}{l}\text { Ventilatory } \\
\text { equivalent }\end{array}$} & \multicolumn{2}{|c|}{ Resp. rate } \\
\hline $\begin{array}{l}\text { AN } 1 \\
\text { AN } 2 \\
\text { AN } 3 \\
\text { AN } 4 \\
\text { AH } 5 \\
\text { AN } 6 \\
\text { AN } 7 \\
\text { AN } 8 \\
\text { AH } 38 \\
\text { AH } 39\end{array}$ & $\begin{array}{l}M \\
\text { F } \\
F \\
F \\
F \\
F \\
F \\
F \\
M \\
F\end{array}$ & $\begin{array}{l}14 \\
38 \\
30 \\
16 \\
24 \\
28 \\
34 \\
51 \\
52 \\
59\end{array}$ & $\begin{array}{l}1.65 \\
1.60 \\
1.70 \\
1.50 \\
1.50 \\
1.60 \\
1.60 \\
1.80 \\
2.10 \\
1.90\end{array}$ & $\begin{array}{l}13 \cdot 7 \\
13 \cdot 3 \\
14 \cdot 4 \\
11 \cdot 5 \\
14 \cdot 8 \\
11 \cdot 6 \\
12.9 \\
14 \cdot 0 \\
15 \cdot 3 \\
12 \cdot 8\end{array}$ & $\begin{array}{l}\text { ASD } \\
\text { ASD } \\
\text { ASD } \\
\text { ASD } \\
\text { ASD } \\
\text { ASD } \\
\text { ASD } \\
\text { ASD } \\
\text { ASD, PH } \\
\text { ASD, PH }\end{array}$ & $\begin{array}{l}1 \\
2 \\
3 \mathrm{~A} \\
1 \\
1 \\
1 \\
2 \\
2 \\
3 \mathrm{~A} \\
2\end{array}$ & $\begin{array}{l}240 \\
200 \\
170 \\
160 \\
190 \\
250 \\
140 \\
230 \\
190 \\
270\end{array}$ & $\begin{array}{l}520 \\
370 \\
420 \\
470 \\
450 \\
390 \\
330 \\
790 \\
410\end{array}$ & $\begin{array}{r}5 \cdot 5 \\
10 \cdot 8 \\
5.5 \\
4 \cdot 1 \\
6.0 \\
6.0 \\
6.9 \\
9.6 \\
6.8 \\
7 \cdot 1\end{array}$ & $\begin{array}{l}16 \cdot 0 \\
12 \cdot 3 \\
12 \cdot 6 \\
10 \cdot 0 \\
13 \cdot 7 \\
10 \cdot 0 \\
17 \cdot 8 \\
21 \cdot 2 \\
11 \cdot 9\end{array}$ & $\begin{array}{l}2 \cdot 3 \\
5 \cdot 3 \\
3 \cdot 2 \\
2 \cdot 6 \\
3 \cdot 2 \\
2 \cdot 4 \\
4 \cdot 9 \\
4 \cdot 2 \\
3 \cdot 6 \\
2 \cdot 6\end{array}$ & $\begin{array}{l}3 \cdot 1 \\
3 \cdot 1 \\
3 \cdot 0 \\
2 \cdot 1 \\
3 \cdot 1 \\
2 \cdot 6 \\
5 \cdot 4 \\
2 \cdot 7 \\
2 \cdot 9\end{array}$ & $\begin{array}{l}12 \\
28 \\
20 \\
14 \\
20 \\
16 \\
21 \\
22 \\
15 \\
17\end{array}$ & $\begin{array}{l}18 \\
28 \\
23 \\
16 \\
30 \\
18 \\
25 \\
20 \\
24\end{array}$ \\
\hline $\begin{array}{l}\text { AH } 40 \\
\text { AH } 41\end{array}$ & $\underset{\mathrm{M}}{\mathrm{F}}$ & $\begin{array}{l}59 \\
54\end{array}$ & $\begin{array}{l}1.50 \\
1.90\end{array}$ & $\begin{array}{l}13 \cdot 3 \\
16 \cdot 7\end{array}$ & $\begin{array}{l}\text { AF } \\
\text { ASD, PH } \\
\text { ASD, PH } \\
\text { AF }\end{array}$ & $\begin{array}{l}2 \\
3 \mathrm{~A}\end{array}$ & $\begin{array}{l}180 \\
180\end{array}$ & $\begin{array}{l}450 \\
760\end{array}$ & $\begin{array}{l}5 \cdot 6 \\
4 \cdot 3\end{array}$ & $\begin{array}{l}13 \cdot 4 \\
17 \cdot 7\end{array}$ & $\begin{array}{l}3 \cdot 1 \\
2 \cdot 4\end{array}$ & $\begin{array}{l}3 \cdot 0 \\
2 \cdot 3\end{array}$ & $\begin{array}{l}19 \\
13\end{array}$ & $\begin{array}{l}21 \\
15\end{array}$ \\
\hline $\begin{array}{l}\text { AH } 42 \\
\text { AH } 43 \\
\text { AH } 44 \\
\text { AH } 45\end{array}$ & $\begin{array}{l}\mathrm{M} \\
\mathrm{F} \\
\mathrm{F} \\
\mathrm{F}\end{array}$ & $\begin{array}{l}48 \\
31 \\
39 \\
49\end{array}$ & $\begin{array}{l}1.80 \\
1.35 \\
1.60 \\
1.70\end{array}$ & $\begin{array}{l}16 \cdot 4 \\
10 \cdot 6 \\
13 \cdot 1 \\
15 \cdot 9\end{array}$ & $\begin{array}{l}\text { ASD, PH } \\
\text { ASD, PH } \\
\text { ASD. PH } \\
\text { ASD, PH } \\
\text { MS, AF }\end{array}$ & $\begin{array}{l}3 \mathrm{~A} \\
3 \mathrm{~A} \\
3 \mathrm{~B} \\
3 \mathrm{~A}\end{array}$ & $\begin{array}{l}200 \\
220 \\
200 \\
180\end{array}$ & $\begin{array}{l}870 \\
600 \\
370 \\
400\end{array}$ & $\begin{array}{l}8 \cdot 8 \\
7 \cdot 3 \\
6 \cdot 6 \\
4 \cdot 4\end{array}$ & $\begin{array}{l}25 \cdot 6 \\
14 \cdot 9 \\
12 \cdot 9 \\
15 \cdot 9\end{array}$ & $\begin{array}{l}4 \cdot 4 \\
3 \cdot 3 \\
3 \cdot 3 \\
2 \cdot 5\end{array}$ & $\begin{array}{l}3.0 \\
2.5 \\
3.5 \\
3.9\end{array}$ & $\begin{array}{l}18 \\
25 \\
15 \\
20\end{array}$ & $\begin{array}{l}24 \\
26 \\
26 \\
28\end{array}$ \\
\hline $\begin{array}{ll}\text { AS } & 82 \\
\text { AS } & 83 \\
\text { VN } & 1 \\
\text { VN } & 2 \\
\text { VN } & 3 \\
\text { VN } & 4 \\
\text { VN } & 5 \\
\text { VH } & 31 \\
\text { VH } & 32 \\
\text { VH } & 33 \\
\text { VH } & 34\end{array}$ & $\begin{array}{l}M \\
F \\
M \\
M \\
M \\
F \\
M \\
M \\
M \\
M \\
M\end{array}$ & $\begin{array}{r}22 \\
9 \\
14 \\
16 \\
37 \\
15 \\
15 \\
26 \\
17 \\
18 \\
62\end{array}$ & $\begin{array}{l}1.90 \\
0.90 \\
1.60 \\
1.50 \\
1.80 \\
1.50 \\
1.40 \\
1.80 \\
1.50 \\
1.60 \\
1.80\end{array}$ & $\begin{array}{l}13 \cdot 9 \\
12 \cdot 6 \\
14 \cdot 0 \\
13 \cdot 6 \\
14 \cdot 7 \\
12 \cdot 8 \\
11 \cdot 8 \\
15 \cdot 0 \\
15 \cdot 3 \\
15 \cdot 8 \\
17 \cdot 2\end{array}$ & $\begin{array}{l}\text { ASD, PS } \\
\text { ASD, PS } \\
\text { VSD } \\
\text { VSD } \\
\text { VSD } \\
\text { VSD } \\
\text { VSD } \\
\text { VSD, PH } \\
\text { VSD, PH } \\
\text { VSD, PH } \\
\text { VSD, PH } \\
\text { AF }\end{array}$ & $\begin{array}{l}1 \\
2 \\
2 \\
1 \\
1 \\
1 \\
1 \\
1 \\
2 \\
2 \\
3 \mathrm{~B}\end{array}$ & $\begin{array}{l}280 \\
130 \\
250 \\
180 \\
240 \\
170 \\
230 \\
280 \\
200 \\
230 \\
220\end{array}$ & $\begin{array}{l}590 \\
230 \\
800 \\
780 \\
620 \\
610 \\
440 \\
670 \\
530 \\
470 \\
-\end{array}$ & $\begin{array}{l}7 \cdot 5 \\
4 \cdot 4 \\
6 \cdot 5 \\
5 \cdot 0 \\
5 \cdot 3 \\
4 \cdot 8 \\
6 \cdot 9 \\
6 \cdot 6 \\
5 \cdot 3 \\
6 \cdot 7 \\
8 \cdot 4\end{array}$ & $\begin{array}{r}19.5 \\
9.9 \\
21.3 \\
11.0 \\
16.9 \\
18.2 \\
12.5 \\
17.2 \\
13.9 \\
14.5 \\
-\end{array}$ & $\begin{array}{l}2 \cdot 7 \\
3 \cdot 4 \\
2 \cdot 6 \\
2 \cdot 8 \\
2 \cdot 2 \\
2 \cdot 8 \\
3 \cdot 9 \\
2 \cdot 4 \\
2 \cdot 6 \\
2 \cdot 9 \\
3 \cdot 8\end{array}$ & $\begin{array}{l}3 \cdot 3 \\
4 \cdot 2 \\
2 \cdot 7 \\
1.5 \\
2 \cdot 7 \\
3 \cdot 0 \\
2.9 \\
2 \cdot 6 \\
2 \cdot 6 \\
3.1 \\
-\end{array}$ & $\begin{array}{l}17 \\
20 \\
14 \\
15 \\
16 \\
19 \\
25 \\
15 \\
16 \\
18 \\
15\end{array}$ & $\begin{array}{l}20 \\
30 \\
17 \\
23 \\
22 \\
26 \\
32 \\
26 \\
21 \\
22 \\
-\end{array}$ \\
\hline $\begin{array}{ll}\text { VE } & 62 \\
\text { VS } & 81 \\
\text { VS } & 82 \\
\text { VS } & 83 \\
\text { PN } & 1 \\
\text { PH } & 30 \\
\text { PE } & 65\end{array}$ & $\begin{array}{l}F \\
M \\
M \\
M \\
M \\
F \\
F\end{array}$ & $\begin{array}{l}19 \\
23 \\
19 \\
29 \\
10 \\
21 \\
34\end{array}$ & $\begin{array}{l}1.60 \\
1.90 \\
1.70 \\
1.80 \\
1.20 \\
1.30 \\
1.60\end{array}$ & $\begin{array}{l}12 \cdot 3 \\
15 \cdot 8 \\
15 \cdot 6 \\
15 \cdot 1 \\
11 \cdot 7 \\
13 \cdot 4 \\
15 \cdot 1\end{array}$ & $\begin{array}{l}\text { VSD, PH } \\
\text { VSD, PS } \\
\text { VSD, PS } \\
\text { VSD, PS } \\
\text { PDA } \\
\text { PDA, PH } \\
\text { PDA, PH }\end{array}$ & $\begin{array}{l}3 \mathrm{~A} \\
1 \\
2 \\
2 \\
1 \\
2 \\
2\end{array}$ & $\begin{array}{l}210 \\
250 \\
250 \\
210 \\
160 \\
180 \\
160\end{array}$ & $\begin{array}{l}550 \\
560 \\
660 \\
-750 \\
320 \\
610\end{array}$ & $\begin{array}{l}7 \cdot 0 \\
6 \cdot 6 \\
7 \cdot 0 \\
6 \cdot 2 \\
4 \cdot 1 \\
6 \cdot 6 \\
5 \cdot 5\end{array}$ & $\begin{array}{l}15 \cdot 5 \\
12 \cdot 0 \\
17 \cdot 6 \\
12 \cdot 5 \\
14 \cdot 3 \\
18 \cdot 9\end{array}$ & $\begin{array}{l}3 \cdot 3 \\
2 \cdot 6 \\
2 \cdot 8 \\
3 \cdot 0 \\
2 \cdot 6 \\
3 \cdot 7 \\
3 \cdot 4\end{array}$ & $\begin{array}{l}2 \cdot 8 \\
2 \cdot 1 \\
2 \cdot 7 \\
2 \cdot 8 \\
4 \cdot 5 \\
3 \cdot 1\end{array}$ & $\begin{array}{l}14 \\
15 \\
15 \\
14 \\
15 \\
30 \\
18\end{array}$ & $\begin{array}{l}20 \\
20 \\
22 \\
17 \\
36 \\
21\end{array}$ \\
\hline
\end{tabular}

ASD, atrial septal defect; VSD, ventricular septal defect; MS, mitral stenosis; PS, pulmonary stenosis; PH, pulmonary hypertension; $A F$, atrial fibrillation; PDA, patent ductus arteriosus.

STPD, standard temperature $\left(37^{\circ} \mathrm{C}\right.$.), pressure $(760 \mathrm{~mm}$. Hg), dry; BTPS, body temperature and pressure, saturated with water vapour. Clinical disability according to New York Heart Association (1953); Grade 3 divided into 3 A and 3 B after Donald, Bishop, and Wade (1954).

uptake against the pulmonary arterial pressure. Some degree of hyperventilation was present in most patients irrespective of the pressure. Similar results are seen when excess ventilation is related to the magnitude of the pulmonary flow or to the left-to-right shunt.

Figure 3 shows the deviation of exercise ventilation from normal against the degree of impairment of cardiac output, i.e. deviation from mean predicted value for the given oxygen uptake (Gazetopoulos et al., 1966b). It appears that the systemic flow is not a determinant of hyperventilation.

No single hæmodynamic parameter is thus associated clearly with the tendency to hyperventilate, which is seen in most of these patients. Other parameters such as pulmonary vascular resistance and the product of pulmonary arterial pressure and flow were examined, but again no close relationship with ventilation was found. It must be noted that these observations apply to low exercise loads in the supine position.
Ventilation and Arterial and Venous Blood Chemistry. Figures 4 and 5 show the arterial $\mathrm{PcO}_{2}$ and $p \mathrm{H}$, measured in the second half of exercise, plotted against the excess ventilation. A number of patients is seen to be hypocapnic, in accordance with their tendency to hyperventilate. Despite this, the $p \mathrm{H}$ in most cases fell owing to lactic acidæmia. Fig. 6 shows the arterial lactate concentration and the excess ventilation: there is some suggestion that the patients with the higher lactates tend to have a greater ventilation, but the relation is not significant $(\mathrm{p}>0 \cdot 1)$.

Figures 7 and 8 deal with the blood gases on the venous side of the circulation. Fig. 7 illustrates the excess ventilation and the pulmonary arterial $p H$. No relation is seen. In Fig. 8 we have plotted the pulmonary arterial $\mathrm{Pco}_{2}$ and the minute ventilation. The broken line represents a normal ventilation-mixed venous $\mathrm{PcO}_{2}$ curve taken from Riley et al. (1963). In the same figure we have plotted the findings in other forms of heart disease 
TABLE II BLOOD CHEMISTRY AT REST AND ON EXERCISE DURING CARDIAC CATHETERIZATION

\begin{tabular}{|c|c|c|c|c|c|c|c|c|c|c|c|}
\hline \multirow{2}{*}{\multicolumn{2}{|c|}{ Case No. }} & \multicolumn{6}{|c|}{ Arterial blood } & \multicolumn{4}{|c|}{ Pulmonary arterial blood } \\
\hline & & $\mathrm{So}_{2}$ & $p \mathrm{H}$ & $\begin{array}{c}\mathrm{Pco}_{2} \\
(\mathrm{~mm} . \mathrm{Hg})\end{array}$ & $\begin{array}{c}\text { Bicarb. } \\
\text { (mEq/l.) }\end{array}$ & $\begin{array}{l}\text { Lactate } \\
(\mathrm{mM} / 1 .)\end{array}$ & $\begin{array}{c}\text { Pyruvate } \\
\text { (mM/1.) }\end{array}$ & $\mathrm{So}_{2}$ & $p \mathrm{H}$ & $\begin{array}{c}\mathrm{Pco}_{2} \\
(\mathrm{~mm} . \mathrm{Hg})\end{array}$ & $\begin{array}{c}\text { Bicarb. } \\
(\mathrm{mEq} / \mathrm{i} .)\end{array}$ \\
\hline AN 1 & $\begin{array}{l}\mathbf{R} \\
\mathbf{E}\end{array}$ & $\begin{array}{l}97 \cdot 8 \\
97 \cdot 1\end{array}$ & $\begin{array}{l}7.420 \\
7 \cdot 410\end{array}$ & $\begin{array}{l}33 \cdot 0 \\
35 \cdot 0\end{array}$ & $\begin{array}{l}21 \cdot 5 \\
22 \cdot 0\end{array}$ & $\frac{1.23}{1.76}$ & $\begin{array}{l}0.11 \\
0.12\end{array}$ & $\begin{array}{l}88.5 \\
80 \cdot 8\end{array}$ & $\begin{array}{l}7 \cdot 405 \\
7 \cdot 380\end{array}$ & $\begin{array}{l}35 \cdot 0 \\
38.0\end{array}$ & $\begin{array}{r}21 \cdot 5 \\
22 \cdot 0\end{array}$ \\
\hline AN 4 & $\underset{\mathbf{E}}{\mathbf{R}}$ & $\frac{95.0}{94.9}$ & $=$ & $=$ & $=$ & $=$ & $=$ & $\begin{array}{l}87.6 \\
69.5 \\
71.0\end{array}$ & $\begin{array}{l}7.360 \\
7.340 \\
7.350\end{array}$ & $\begin{array}{l}45.0 \\
58.0 \\
56.0\end{array}$ & $\begin{array}{l}23 \cdot 0 \\
23 \cdot 0 \\
24 \cdot 5\end{array}$ \\
\hline AN 5 & $\underset{\mathbf{E}}{\mathbf{R}}$ & $\frac{95 \cdot 1}{94 \cdot 8}$ & $\begin{array}{l}7.415 \\
7 \cdot 395\end{array}$ & $\begin{array}{l}\frac{42 \cdot 0}{41 \cdot 0} \\
\frac{41}{2}\end{array}$ & $\frac{25 \cdot 0}{24 \cdot 0}$ & $\begin{array}{l}0.85 \\
1.11 \\
1.26\end{array}$ & $\begin{array}{l}0.07 \\
0.08 \\
0.08\end{array}$ & $\frac{90.0}{83.6}$ & $\frac{7 \cdot 400}{7 \cdot 375}$ & $\begin{array}{l}\frac{42 \cdot 0}{45 \cdot 0} \\
\frac{15}{4}\end{array}$ & $\begin{array}{l}24 \cdot 5 \\
24 \cdot 0\end{array}$ \\
\hline AN 6 & $\underset{\mathbf{E}}{\mathbf{R}}$ & $\begin{array}{l}96.0 \\
97.0 \\
97.1\end{array}$ & $\begin{array}{l}7.405 \\
7.425 \\
7.410\end{array}$ & $\begin{array}{l}32.5 \\
33.0 \\
36.5\end{array}$ & $\begin{array}{l}22 \cdot 0 \\
23.0 \\
23 \cdot 0\end{array}$ & $\begin{array}{l}0.65 \\
0.72 \\
0.78\end{array}$ & $\begin{array}{l}0.07 \\
0.07 \\
0.10\end{array}$ & $\begin{array}{l}89.0 \\
85.0 \\
87.5\end{array}$ & $\begin{array}{l}7.405 \\
7.415 \\
7.410\end{array}$ & $\begin{array}{l}34 \cdot 5 \\
32.0 \\
37.0\end{array}$ & $\begin{array}{l}22 \cdot 5 \\
21.5 \\
23 \cdot 0\end{array}$ \\
\hline AH 38 & $\underset{\mathbf{E}}{\mathbf{R}}$ & $\begin{array}{l}93 \cdot 4 \\
92.9 \\
89.1\end{array}$ & $\begin{array}{l}7.350 \\
7.350 \\
7.350\end{array}$ & $\begin{array}{l}36 \cdot 0 \\
36.0 \\
34 \cdot 0\end{array}$ & $\begin{array}{l}20.0 \\
20.0 \\
19.5\end{array}$ & $\begin{array}{l}0.91 \\
1.24 \\
1.30\end{array}$ & $\begin{array}{l}0.06 \\
0.07 \\
0.09\end{array}$ & $\begin{array}{l}81 \cdot 9 \\
71.0 \\
63.7\end{array}$ & $\begin{array}{l}7.345 \\
3.330 \\
7.330\end{array}$ & $\begin{array}{l}39.9 \\
40.0 \\
40.0\end{array}$ & $\begin{array}{l}20.0 \\
19.5 \\
19.5\end{array}$ \\
\hline AH 39 & $\underset{\mathbf{E}}{\mathbf{R}}$ & $\frac{96 \cdot 0}{94 \cdot 5}$ & $\begin{array}{l}7.425 \\
7.430 \\
7.430\end{array}$ & $\begin{array}{l}48 \cdot 0 \\
40 \cdot 0 \\
41 \cdot 0\end{array}$ & $\begin{array}{l}28.5 \\
25.5 \\
26.0\end{array}$ & $\begin{array}{l}0.52 \\
0.72 \\
0.98\end{array}$ & $\begin{array}{l}0.07 \\
0.10 \\
0.09\end{array}$ & $\begin{array}{l}86 \cdot 4 \\
76.5 \\
79 \cdot 6\end{array}$ & $\begin{array}{l}7.415 \\
7.425 \\
7.415\end{array}$ & $\begin{array}{l}49.0 \\
46.0 \\
52.0\end{array}$ & $\begin{array}{l}26 \cdot 5 \\
27.0 \\
27 \cdot 0\end{array}$ \\
\hline AH 40 & $\begin{array}{l}\mathbf{R} \\
\mathbf{E}\end{array}$ & $\begin{array}{l}94.6 \\
92.2 \\
94.6\end{array}$ & $\begin{array}{l}7.360 \\
7.355 \\
-\end{array}$ & $=$ & $=$ & $\begin{array}{l}1.04 \\
1.76 \\
2.80\end{array}$ & $\stackrel{0.13}{=}$ & $\begin{array}{l}87.5 \\
78.0 \\
80.7\end{array}$ & $=$ & $=$ & $=$ \\
\hline AH 41 & $\underset{\mathbf{E}}{\mathbf{R}}$ & $\begin{array}{l}95.9 \\
92.1 \\
92.1\end{array}$ & $\begin{array}{l}7.380 \\
7.375 \\
7.330\end{array}$ & $\begin{array}{l}39.5 \\
39.5 \\
41.0\end{array}$ & $\begin{array}{l}22 \cdot 5 \\
22.5 \\
22.5\end{array}$ & $\begin{array}{l}0.72 \\
1.30 \\
1.89\end{array}$ & $\begin{array}{l}0.11 \\
0.12 \\
0.14\end{array}$ & $\begin{array}{l}83 \cdot 2 \\
70.7 \\
73 \cdot 0\end{array}$ & $\begin{array}{l}7.365 \\
7.365 \\
7.345\end{array}$ & $\begin{array}{l}44.0 \\
46.0 \\
48.0\end{array}$ & $\begin{array}{l}23.5 \\
22.0 \\
22.0\end{array}$ \\
\hline AH 42 & $\underset{\mathbf{E}}{\mathbf{R}}$ & $\begin{array}{l}91 \cdot 6 \\
93.7 \\
90.0\end{array}$ & $\begin{array}{l}7.390 \\
7.400 \\
7.370\end{array}$ & $\begin{array}{l}35.0 \\
31.0 \\
34.5\end{array}$ & $\begin{array}{l}22.4 \\
21.0 \\
20.5\end{array}$ & $\begin{array}{l}1.04 \\
2.20 \\
2.14\end{array}$ & $\begin{array}{l}0.08 \\
0.12 \\
0.13\end{array}$ & $\begin{array}{l}82.5 \\
71.4 \\
71.7\end{array}$ & $\begin{array}{l}7.370 \\
7.370 \\
7.365\end{array}$ & $\begin{array}{l}38.0 \\
38.0 \\
38.5\end{array}$ & $\begin{array}{l}21 \cdot 0 \\
20.5 \\
20.5\end{array}$ \\
\hline AH 43 & $\underset{\mathbf{E}}{\mathbf{R}}$ & $\begin{array}{l}92.6 \\
89.8 \\
88.1\end{array}$ & $\begin{array}{l}7.415 \\
7.370 \\
7.345\end{array}$ & $\begin{array}{l}35.0 \\
37.5 \\
37.5\end{array}$ & $\begin{array}{l}23 \cdot 0 \\
21.0 \\
20 \cdot 0\end{array}$ & $\begin{array}{l}0.78 \\
2.14 \\
2.90\end{array}$ & $\begin{array}{l}0.06 \\
0.12 \\
0.16\end{array}$ & $\begin{array}{l}86.5 \\
75.6 \\
76.7\end{array}$ & $=$ & $=$ & 三 \\
\hline AH 45 & $\underset{\mathbf{E}}{\mathbf{R}}$ & $\begin{array}{l}93 \cdot 7 \\
95 \cdot 1\end{array}$ & $\begin{array}{l}7.390 \\
7.390\end{array}$ & $\frac{38.0}{37.5}$ & $\frac{23 \cdot 0}{23 \cdot 0}$ & $\begin{array}{l}0.98 \\
1.89\end{array}$ & $\frac{0.09}{0.10}$ & $\begin{array}{l}87.0 \\
87.0\end{array}$ & $\begin{array}{l}7 \cdot 380 \\
7 \cdot 345\end{array}$ & $\begin{array}{l}40.0 \\
40.5\end{array}$ & $\frac{22 \cdot 5}{21 \cdot 0}$ \\
\hline VN 2 & $\underset{\mathbf{E}}{\mathbf{R}}$ & $\begin{array}{l}98 \cdot 4 \\
95 \cdot 6\end{array}$ & $\stackrel{7 \cdot 400}{=}$ & $\stackrel{40 \cdot 0}{=}$ & $\stackrel{24 \cdot 0}{=}$ & 三 & $=$ & $\frac{78.0}{68.3}$ & $\frac{7 \cdot 395}{7 \cdot 370}$ & $\begin{array}{l}40 \cdot 0 \\
40 \cdot 0\end{array}$ & $\frac{24.0}{22.5}$ \\
\hline VN 3 & $\underset{\mathbf{E}}{\mathbf{R}}$ & $\begin{array}{l}94 \cdot 3 \\
92 \cdot 9\end{array}$ & $=$ & $=$ & 三 & $\bar{z}$ & $=$ & $\begin{array}{l}81 \cdot 0 \\
68.1 \\
68.5\end{array}$ & $\begin{array}{l}7.370 \\
7.350 \\
7.345\end{array}$ & $\begin{array}{l}52.0 \\
53.0 \\
58.5\end{array}$ & $\begin{array}{l}26.0 \\
25.0 \\
25 \cdot 0\end{array}$ \\
\hline VN 5 & $\underset{\mathbf{E}}{\mathbf{R}}$ & $\frac{98 \cdot 0}{97 \cdot 2}$ & $\begin{array}{l}7.410 \\
7 \cdot 440\end{array}$ & $\begin{array}{l}32 \cdot 5 \\
30 \cdot 0\end{array}$ & $\frac{21.5}{22.5}$ & $\frac{0.70}{1 \cdot 30}$ & $\frac{0.09}{0.12}$ & $\stackrel{90.2}{=}$ & $\stackrel{7.370}{=}$ & $\begin{array}{l}38.5 \\
=\end{array}$ & $\stackrel{22 \cdot 0}{=}$ \\
\hline VH 31 & $\frac{\mathbf{R}}{\mathbf{E}}$ & $\begin{array}{l}98.5 \\
95.7\end{array}$ & $\begin{array}{l}7.350 \\
7.345\end{array}$ & $\begin{array}{l}48 \cdot 0 \\
47.5\end{array}$ & $\frac{24 \cdot 0}{23 \cdot 0}$ & $\begin{array}{l}0.96 \\
1.43 \\
1.78\end{array}$ & $\begin{array}{l}0.10 \\
0.11 \\
0.13\end{array}$ & $\begin{array}{l}86.9 \\
78.8\end{array}$ & $\begin{array}{l}7 \cdot 310 \\
7 \cdot 310\end{array}$ & $\frac{51.5}{56.5}$ & $\frac{22 \cdot 6}{22 \cdot 4}$ \\
\hline VH 32 & $\underset{\mathbf{E}}{\mathbf{R}}$ & $\begin{array}{l}98.6 \\
97.5\end{array}$ & $\begin{array}{l}7.370 \\
7 \cdot 385\end{array}$ & $\begin{array}{l}\frac{44 \cdot 0}{36 \cdot 5} \\
\text {. }\end{array}$ & $\frac{23.8}{22.0}$ & $\begin{array}{l}0.86 \\
0.94 \\
1.22\end{array}$ & $\begin{array}{l}0.09 \\
0.10 \\
0.09\end{array}$ & $\frac{90 \cdot 0}{83 \cdot 2}$ & $\begin{array}{l}7.335 \\
7 \cdot 345\end{array}$ & $\frac{50 \cdot 0}{52 \cdot 5}$ & $\frac{23 \cdot 3}{23 \cdot 8}$ \\
\hline VH 33 & $\underset{\mathbf{E}}{\mathbf{R}}$ & $\begin{array}{l}94 \cdot 5 \\
92 \cdot 2\end{array}$ & $\begin{array}{l}7.385 \\
7.380 \\
7.380\end{array}$ & $\begin{array}{l}34.0 \\
38.5 \\
35.5\end{array}$ & $\begin{array}{l}20.5 \\
22.0 \\
21.5\end{array}$ & $=$ & $\begin{array}{l}0.12 \\
0.12 \\
0.17\end{array}$ & $\begin{array}{l}88.4 \\
68.4 \\
68.5\end{array}$ & $\begin{array}{l}7.380 \\
7.335 \\
7 \cdot 340\end{array}$ & $\begin{array}{l}36.0 \\
46.0 \\
39.0\end{array}$ & $\begin{array}{l}21.0 \\
22.0 \\
19.5\end{array}$ \\
\hline VE 62 & $\begin{array}{l}\mathbf{R} \\
\mathbf{E}\end{array}$ & $\begin{array}{l}91.8 \\
82.9 \\
85.0\end{array}$ & $\begin{array}{l}7.425 \\
7.415 \\
\mathbf{7 . 4 1 0}\end{array}$ & $\begin{array}{l}33.0 \\
34.5 \\
33.0\end{array}$ & $\begin{array}{l}23 \cdot 0 \\
23 \cdot 0 \\
22 \cdot 0\end{array}$ & $\begin{array}{l}0.97 \\
2.08 \\
1.57\end{array}$ & $\begin{array}{l}0.07 \\
0.11 \\
0.12\end{array}$ & $\begin{array}{l}77.6 \\
68.5 \\
63.5\end{array}$ & $\begin{array}{l}7.400 \\
7.370 \\
7 \cdot 375\end{array}$ & $\begin{array}{l}36 \cdot 0 \\
39 \cdot 0 \\
38.5\end{array}$ & $\begin{array}{l}22 \cdot 0 \\
21 \cdot 0 \\
21 \cdot 0\end{array}$ \\
\hline VS 81 & $\underset{\mathbf{E}}{\mathbf{R}}$ & $\begin{array}{l}97.9 \\
97.9\end{array}$ & $\begin{array}{l}7 \cdot 330 \\
7 \cdot 370\end{array}$ & $\frac{44 \cdot 2}{39 \cdot 0}$ & $\frac{24 \cdot 0}{23 \cdot 4}$ & $\begin{array}{l}0.59 \\
0.84 \\
0.88\end{array}$ & $\begin{array}{l}0.06 \\
0.07 \\
0.08\end{array}$ & $\begin{array}{l}88 \cdot 3 \\
85 \cdot 0\end{array}$ & $\overline{\overline{7} \cdot 330}$ & $\overline{44 \cdot 0}$ & $\overline{23.5}$ \\
\hline vS 82 & $\underset{\mathbf{E}}{\mathbf{R}}$ & $\begin{array}{l}96.0 \\
92.0\end{array}$ & $\begin{array}{r}7 \cdot 440 \\
7 \cdot 455\end{array}$ & $\frac{42.0}{37.0}$ & $\frac{27 \cdot 0}{26 \cdot 0}$ & $\begin{array}{l}0.68 \\
1.50\end{array}$ & $\begin{array}{l}0.11 \\
0.12\end{array}$ & $\frac{82 \cdot 8}{71 \cdot 7}$ & 三 & 二 & $=$ \\
\hline PN 1 & $\begin{array}{l}\mathbf{R} \\
\mathbf{E}\end{array}$ & $\begin{array}{l}\frac{97 \cdot 4}{97 \cdot 2} \\
\end{array}$ & $\begin{array}{l}7 \cdot 285 \\
7 \cdot 315\end{array}$ & $\begin{array}{r}42.5 \\
38.0\end{array}$ & $\frac{19 \cdot 0}{21 \cdot 0}$ & $\begin{array}{l}0.65 \\
1.20 \\
1.04\end{array}$ & $\begin{array}{l}0.09 \\
0.11 \\
0.12\end{array}$ & $\stackrel{78.4}{=}$ & $\stackrel{7.270}{=}$ & $\stackrel{48 \cdot 0}{=}$ & $\stackrel{20 \cdot 0}{=}$ \\
\hline
\end{tabular}


TABLE II-continued

\begin{tabular}{|c|c|c|c|c|c|c|c|c|c|c|c|}
\hline \multirow[b]{2}{*}{ Case No. } & & \multicolumn{6}{|c|}{ Arterial blood } & \multicolumn{4}{|c|}{ Pulmonary arterial blood } \\
\hline & & $\mathrm{So}_{2}$ & $p \mathrm{H}$ & $\begin{array}{c}\mathrm{Pco}_{2} \\
(\mathrm{~mm} . \mathrm{Hg})\end{array}$ & $\begin{array}{l}\text { Bicarb. } \\
\text { (mEq/l.) }\end{array}$ & $\begin{array}{l}\text { Lactate } \\
(\mathrm{mM} / 1 .)\end{array}$ & $\begin{array}{l}\text { Pyruvate } \\
(\mathrm{mM} / \mathrm{l} .)\end{array}$ & $\mathrm{So}_{2}$ & pH & $\underset{(\mathrm{mm} . \mathrm{Hg})}{\mathrm{PcO}_{2}}$ & $\underset{(\mathrm{mEq} / \mathrm{l} .)}{\text { Bicarb. }}$ \\
\hline PH 30 & $\begin{array}{l}\mathbf{R} \\
\mathbf{E}\end{array}$ & $\frac{95.5}{95.0}$ & 二 & $=$ & $=$ & $\frac{0.91}{1.50}$ & $\frac{0.06}{0.09}$ & $\begin{array}{l}90 \cdot 2 \\
78 \cdot 6 \\
75 \cdot 8\end{array}$ & 二 & 二 & 二 \\
\hline PE 65 & $\begin{array}{l}\mathbf{R} \\
\mathbf{E}\end{array}$ & $\begin{array}{l}93.5 \\
90.0 \\
89.9\end{array}$ & $\begin{array}{l}7 \cdot 415 \\
7 \cdot 370 \\
7 \cdot 360\end{array}$ & $\begin{array}{l}38 \cdot 0 \\
40 \cdot 5 \\
38 \cdot 0\end{array}$ & $\begin{array}{l}24 \cdot 0 \\
22 \cdot 0 \\
20 \cdot 0\end{array}$ & $\begin{array}{l}0.53 \\
1.89 \\
2.54\end{array}$ & $\begin{array}{l}0.07 \\
0.11 \\
0.12\end{array}$ & $=$ & $\bar{z}$ & 三 & $\bar{z}$ \\
\hline
\end{tabular}

Note: The first exercise sample was obtained in the 3 rd and 4 th minute, the second between the 8 th and 10 th minutes.

$R$, rest; $E$, exercise; $\mathrm{So}_{2}$, oxygen saturation.

without septal defects, shown by crosses (Gazetopoulos et al., 1966a). It is seen that the higher the ventilation, the lower the pulmonary arterial $\mathrm{Pco}_{2}$.

It appears therefore that in these circumstances neither the arterial nor the venous blood chemistry exerts an influence on the ventilation.

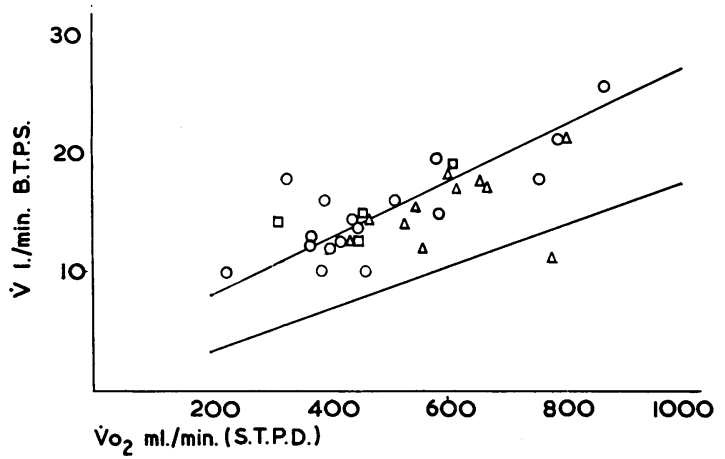

Fig. 1.-Ventilation ana oxygen uptake during exercise in the supine position at cardiac catheterization. The normal limits are shown. $O$ atrial septal defect; $\Delta$ ventricular septal defect; $\square$ patent ductus arteriosus.

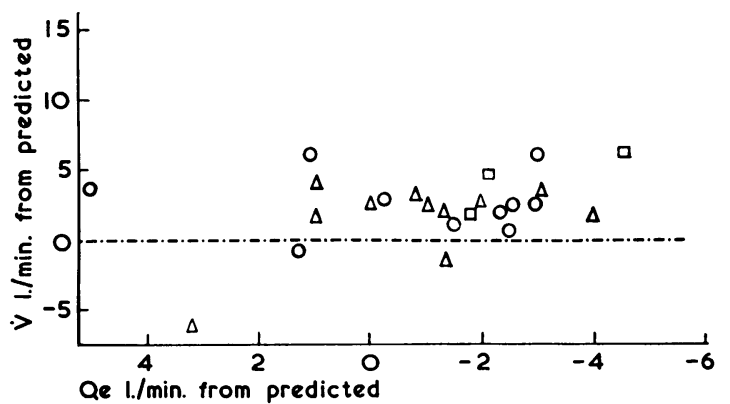

Fig. 3.-Excess ventilation in relation to the degree of impairment of cardiac output, expressed as deviation from the mean predicted for the given oxygen uptake. Symbols and definitions as in Fig. 1 and 2.
Upright Exercise. Table III shows the findings in subjects exercised in the upright position on the bicycle ergometer with stepwise increases in load. Fig. 9 illustrates the main parameters studied: in the lower part the ventilation and heart-rate in the steady state are shown, the normal limits (Davies

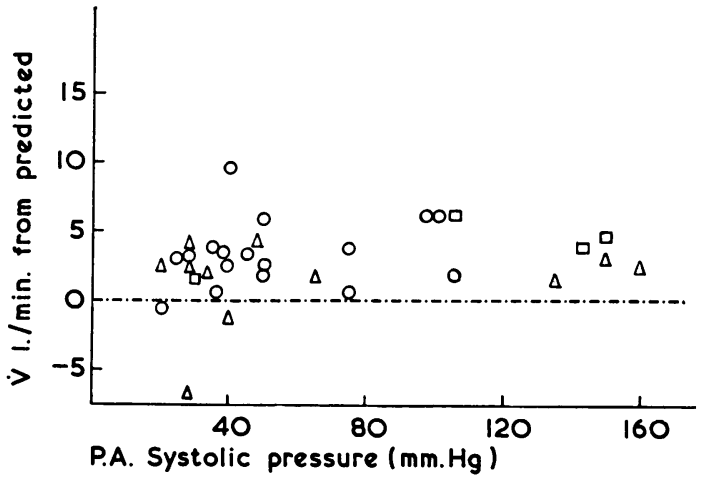

FIG. 2.-Ventilation during supine exercise expressed as deviation from the mean predicted normal for the given oxygen uptake, in relation to pulmonary arterial systolic pressure. Symbols as in Fig. 1.

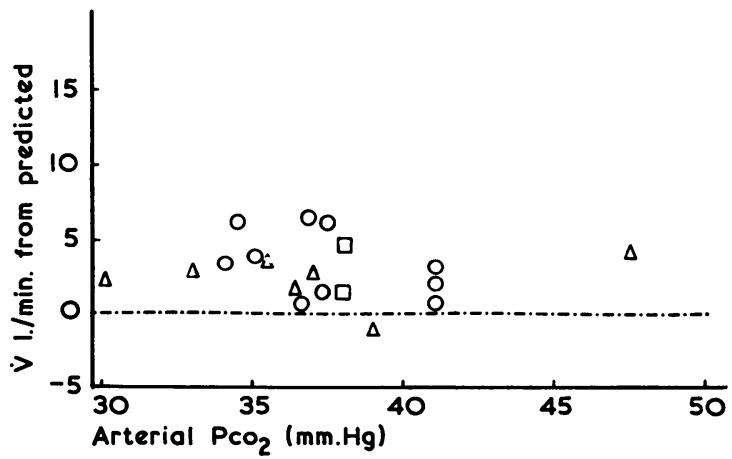

FIG. 4.-Excess ventilation and arterial $\mathrm{Pco}_{2}$. Symbols and definitions as in Fig. 1 and 2 . 


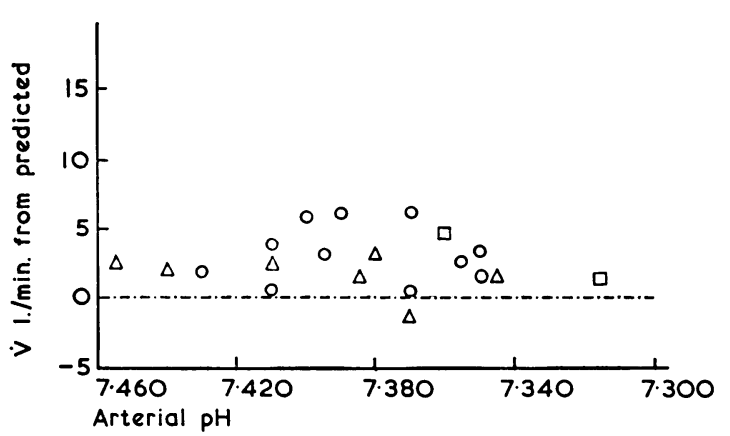

FIG. 5.-Excess ventilation and arterial $p \mathrm{H}$. Symbols and definitions as in Fig. 1 and 2.

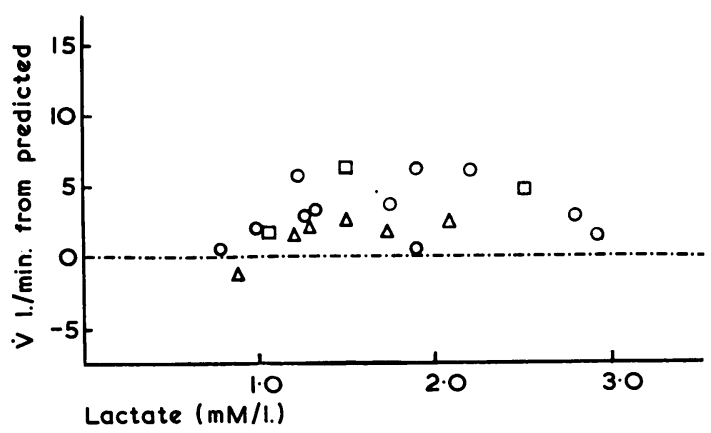

FIG. 6.-Excess ventilation and arterial lactate. Symbols and definitions as in Fig 1 and 2.

et al., 1965) being also indicated. Above them are given the $p \mathrm{H}, \mathrm{Pco}_{2}$, oxygen saturation, and lactate concentration at the end of each 10-minute period. Four of the patients (Cases AN5, AH39, AH45, and VH 34) were able to perform at only lower levels $(200-400 \mathrm{kpm} . / \mathrm{min}$.$) , and in all these dyspnœa$

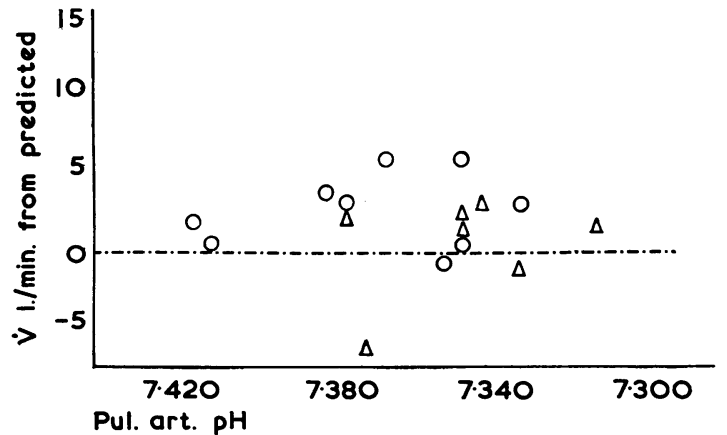

FIG. 7.-Excess ventilation and pulmonary arterial $p \mathbf{H}$. Symbols and definitions as in Fig. 1 and 2.

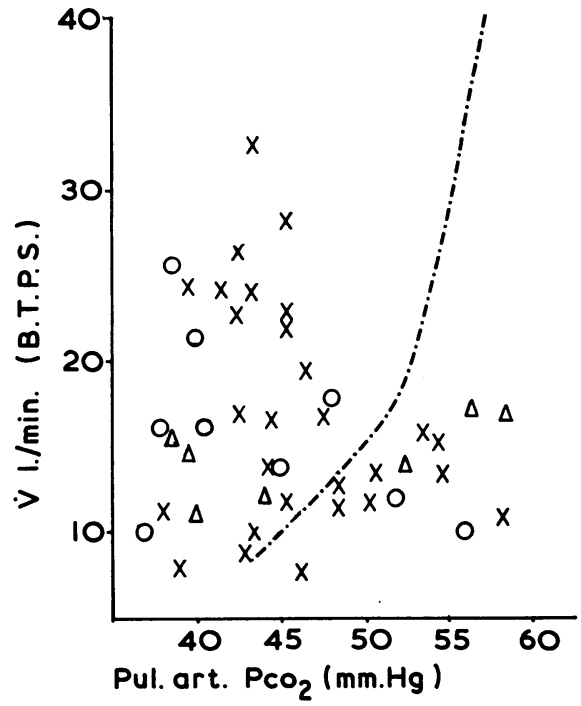

Fig. 8.-Ventilation and pulmonary arterial $\mathrm{Pco}_{2}$. Xs represent patients without septal defects (Gazetopoulos et al., 1966a). Otherwise symbols as in Fig. 1. The broken line represents the normal $\dot{\mathrm{V}}$-mixed venous $\mathrm{Pco}_{2}$ relationship from Riley et al. (1963). High ventilation is associated with lower values of $\mathrm{PCO}_{2}$.

was the limiting factor. Ventilation was excessive, and the blood chemistry showed changes consequent on hyperventilation. In two of them (Cases AH45 and VH32) raised pulmonary venous pressure could have been a stimulus to ventilation but in the other two it was normal. Both these patients (Cases AN5 and AH39) showed severe respiratory embarrassment after surgery, as a result of which they died. We have at present no explanation for this.

The other patients were able to reach higher exercise loads, and in many of them fatigue rather than dyspnœa appeared to be the limiting factor while hyperventilation was less obvious or not present. We note that some patients with high pulmonary arterial pressure and increased pulmonary blood flow were able to reach moderate exercise loads $(600 \mathrm{kpm} . / \mathrm{min}$.) without impressive hyperventilation. Direct measurements of pressure and flow during upright exercise were not made by us, but the evidence of Bruce and John (1957) suggests that the upright posture does not lead to diminution in pulmonary flow or pressure in such patients, and the findings during supine exercise may reasonably be applied also in the upright position. Pulmonary plæonæmia and hypertension would, therefore, not appear to be essential primary determinants of hyperventilation, even up to maximal exercise tolerance. In all 
TABLE III

FINDINGS BEFORE AND DURING EXERCISE IN THE UPRIGHT POSITION WITH STEPWISE INCREASE OF LOAD

\begin{tabular}{|c|c|c|c|c|c|c|c|c|c|c|c|}
\hline & & Exercise & & & & & & Arterial & blood & & \\
\hline Case Nc & & $\underset{\text { min.) }}{\text { (kpm./ }}$ & $\begin{array}{l}\text { (ml./min.) } \\
\text { STPD }\end{array}$ & $\underset{\text { rate }}{\text { Heart }}$ & $\begin{array}{l}\text { (l./min.) } \\
\text { BTPS }\end{array}$ & $\mathrm{So}_{2}$ & $p H$ & $\underset{(\mathrm{mm} . \mathrm{Hg})}{\mathrm{PcO}_{2}}$ & $\begin{array}{l}\text { Bicarb. } \\
\text { (mEq/l.) }\end{array}$ & $\begin{array}{l}\text { Lactate } \\
(\mathrm{mM} / 1 .)\end{array}$ & $\begin{array}{c}\text { Pyruvate } \\
(\mathrm{mM} / 1 .)\end{array}$ \\
\hline VS 83 & $\mathbf{R}$ & 0 & 306 & 84 & 8.5 & $97 \cdot 0$ & $7 \cdot 455$ & 40.9 & $27 \cdot 0$ & 0.98 & $0 \cdot 10$ \\
\hline & Ex. & 200 & 780 & 112 & $15 \cdot 0$ & $\overline{0.5}$ & $7 \cdot 440$ & $42 \cdot 0$ & $27 \cdot 0$ & 2.01 & 0.12 \\
\hline & & 400 & 1050 & 120 & $21 \cdot 5$ & 90.0 & $\begin{array}{l}7.440 \\
7.440\end{array}$ & 39.0 & $\begin{array}{l}20.5 \\
25.5\end{array}$ & $\begin{array}{l}1.95 \\
2.15\end{array}$ & $\begin{array}{l}0.13 \\
0.14\end{array}$ \\
\hline & & 600 & 1300 & 144 & $29 \cdot 0$ & 98.5 & $\begin{array}{l}7 \cdot 435 \\
7 \cdot 425\end{array}$ & $\begin{array}{l}30.0 \\
34.0\end{array}$ & $\begin{array}{l}24.5 \\
24.5\end{array}$ & $\begin{array}{l}2.47 \\
3.64\end{array}$ & $\begin{array}{l}0.15 \\
0.14\end{array}$ \\
\hline & & 800 & 1700 & 164 & $34 \cdot 0$ & $\begin{array}{l}97 \cdot 0 \\
97 \cdot 2 \\
97 \cdot 0\end{array}$ & $\begin{array}{l}7 \cdot 395 \\
7 \cdot 415 \\
7 \cdot 410\end{array}$ & $\begin{array}{l}40 \cdot 0 \\
38 \cdot 0 \\
36 \cdot 0\end{array}$ & $\begin{array}{l}23 \cdot 5 \\
24 \cdot 0 \\
23 \cdot 0\end{array}$ & $\begin{array}{l}5.20 \\
6.90 \\
7 \cdot 55\end{array}$ & $\begin{array}{l}0.19 \\
0.19 \\
0.19\end{array}$ \\
\hline & $\begin{array}{r}\text { Rec. }\left(5^{\prime}\right) \\
\left(10^{\prime}\right)\end{array}$ & & 二 & $\begin{array}{l}108 \\
104\end{array}$ & = & $\stackrel{97 \cdot 0}{-}$ & $\begin{array}{l}7 \cdot 400 \\
7 \cdot 455\end{array}$ & $\begin{array}{l}36 \cdot 0 \\
34 \cdot 0\end{array}$ & $\begin{array}{l}22.0 \\
24.5\end{array}$ & $\begin{array}{l}6.20 \\
5.65\end{array}$ & $\begin{array}{l}0.26 \\
0.25\end{array}$ \\
\hline AS 82 & $\mathbf{R}$ & 0 & - & 86 & $9 \cdot 3$ & $97 \cdot 0$ & $7 \cdot 420$ & $36 \cdot 2$ & $23 \cdot 0$ & 0.65 & 0.09 \\
\hline & Ex. & 200 & - & 108 & $19 \cdot 5$ & $\overline{97 \cdot 0}$ & $7 \cdot \overline{415}$ & $\overline{34 \cdot 0}$ & $\overline{22 \cdot 0}$ & $\overline{1.43}$ & $\overline{0.12}$ \\
\hline & & 400 & - & 115 & $28 \cdot 2$ & $97 \cdot 0$ & $\begin{array}{l}7 \cdot 415 \\
7 \cdot 410\end{array}$ & $\begin{array}{l}34.0 \\
35.5\end{array}$ & $\begin{array}{l}22 \cdot 0 \\
22 \cdot 0\end{array}$ & $\begin{array}{l}1.43 \\
1.69\end{array}$ & $\begin{array}{l}0.12 \\
0.13\end{array}$ \\
\hline & & 600 & - & 126 & $35 \cdot 8$ & 96.5 & $\begin{array}{l}7 \cdot 415 \\
7 \cdot 420\end{array}$ & $\begin{array}{l}34.0 \\
34.0\end{array}$ & $\begin{array}{l}22 \cdot 0 \\
225\end{array}$ & $\begin{array}{l}2.02 \\
1.56\end{array}$ & $\begin{array}{l}0.14 \\
0.14\end{array}$ \\
\hline & & 800 & - & 160 & $49 \cdot 4$ & 96.5 & $\begin{array}{l}7 \cdot 420 \\
7 \cdot 420\end{array}$ & $\begin{array}{l}33.5 \\
35.0\end{array}$ & $\begin{array}{l}22 \cdot 0 \\
22 \cdot 0\end{array}$ & $\begin{array}{l}2.08 \\
2.34\end{array}$ & $\begin{array}{l}0.14 \\
0.16\end{array}$ \\
\hline & & 1000 & - & 175 & - & $\begin{array}{l}90.3 \\
96.5 \\
-\end{array}$ & $7 \cdot 360$ & 26.5 & 17.0 & $\begin{array}{l}2 \cdot 13 \\
-\end{array}$ & $\begin{array}{l}0.26 \\
-\end{array}$ \\
\hline & $\operatorname{Rec} . \begin{array}{l}\left(5^{\prime}\right) \\
\left(15^{\prime}\right)\end{array}$ & & $=$ & $\begin{array}{l}130 \\
107\end{array}$ & 13.9 & z & $7 \cdot \overline{420}$ & $\overline{34 \cdot 0}$ & $\overline{22 \cdot 0}$ & $\begin{array}{l}5.07 \\
1.36\end{array}$ & 二 \\
\hline PH 30 & $\mathbf{R}$ & 0 & 200 & 96 & 4.0 & - & $7 \cdot 420$ & $34 \cdot 0$ & $22 \cdot 5$ & 0.65 & 0.07 \\
\hline & Ex. & 200 & 560 & 104 & 13.4 & - & $\overline{750}$ & $\overline{20}$ & $\overline{0}$ & - & - \\
\hline & & 400 & 1025 & 140 & $21 \cdot 0$ & $=$ & $\begin{array}{l}7.450 \\
7.400\end{array}$ & $\begin{array}{l}32 \cdot 3 \\
34 \cdot 5 \\
33 \cdot 0\end{array}$ & $\begin{array}{l}23 \cdot 0 \\
22 \cdot 0 \\
22 \cdot 0\end{array}$ & $\begin{array}{l}1.04 \\
2.21 \\
2.03\end{array}$ & $\begin{array}{l}0.08 \\
0.11 \\
0.13\end{array}$ \\
\hline & & 600 & - & 160 & - & 二 & $7 \cdot 430$ & 32.5 & 22.5 & $2 \cdot 37$ & 0.12 \\
\hline AH 45 & $\mathbf{R}$ & 0 & 256 & 80 & $6 \cdot 1$ & $98 \cdot 0$ & $7 \cdot 485$ & 30.5 & $23 \cdot 0$ & 1.36 & 0.06 \\
\hline & Ex. & 200 & 660 & 156 & $19 \cdot 6$ & $\overline{97 \cdot 6}$ & $\begin{array}{l}7 \cdot 500 \\
7 \cdot 450\end{array}$ & $\begin{array}{l}27 \cdot 5 \\
24 \cdot 5\end{array}$ & $\begin{array}{l}22 \cdot 0 \\
19 \cdot 0\end{array}$ & $\begin{array}{l}1.75 \\
6.37\end{array}$ & $\begin{array}{l}0.09 \\
0.13\end{array}$ \\
\hline & & 400 & - & 180 & $34 \cdot 2$ & 98.0 & $\begin{array}{l}7.445 \\
7.500\end{array}$ & $\begin{array}{l}23.5 \\
19.5\end{array}$ & $\begin{array}{l}19.5 \\
18 \cdot 0\end{array}$ & $\begin{array}{l}5 \cdot 46 \\
7 \cdot 54\end{array}$ & $\begin{array}{l}0.16 \\
0 \cdot 17\end{array}$ \\
\hline & Rec. $\begin{array}{r}\left(5^{\prime}\right) \\
\left(15^{\prime}\right)\end{array}$ & & 二 & $\begin{array}{r}112 \\
96\end{array}$ & $\begin{array}{l}6 \cdot 3 \\
4 \cdot 9\end{array}$ & $=$ & $\begin{array}{l}7 \cdot 420 \\
7 \cdot 440\end{array}$ & $\begin{array}{l}26 \cdot 0 \\
26 \cdot 0\end{array}$ & $\begin{array}{l}18 \cdot 5 \\
19,5\end{array}$ & $\begin{array}{l}5 \cdot 85 \\
4.55\end{array}$ & 二 \\
\hline AH 39 & $\mathbf{R}$ & 0 & 265 & 80 & $12 \cdot 2$ & $94 \cdot 0$ & $7 \cdot 525$ & $34 \cdot 5$ & $28 \cdot 5$ & 0.85 & 0.07 \\
\hline & Ex. & 200 & 750 & 128 & $22 \cdot 8$ & $\overline{0}$ & $7 \cdot 500$ & 34.0 & $27 \cdot 5$ & 1.69 & $0 \cdot 10$ \\
\hline & & 400 & 1150 & 156 & $30 \cdot 8$ & $\frac{90.2}{92.0}$ & $\begin{array}{l}7 \cdot 495 \\
7 \cdot 485\end{array}$ & $\begin{array}{l}30 \cdot 0 \\
33 \cdot 0 \\
34 \cdot 0\end{array}$ & $\begin{array}{l}26 \cdot 0 \\
26 \cdot 0\end{array}$ & $\begin{array}{l}1.93 \\
2.86 \\
3.38\end{array}$ & $\begin{array}{l}0.12 \\
0.12 \\
0.13\end{array}$ \\
\hline & Rec. $\begin{array}{l}\left(5^{\prime}\right) \\
\left(10^{\prime}\right)\end{array}$ & $\bar{z}$ & 二 & $\begin{array}{l}100 \\
100\end{array}$ & $\begin{array}{l}15 \cdot 1 \\
11 \cdot 0\end{array}$ & $\overline{96 \cdot 0}$ & $\begin{array}{l}7 \cdot 485 \\
7 \cdot 520\end{array}$ & $\begin{array}{l}32 \cdot 0 \\
30 \cdot 0\end{array}$ & $\begin{array}{l}25 \cdot 0 \\
26 \cdot 0\end{array}$ & $\begin{array}{l}2.47 \\
1.95\end{array}$ & $\begin{array}{l}0 \cdot 15 \\
0 \cdot 13\end{array}$ \\
\hline VH 34 & $\mathbf{R}$ & 0 & 254 & 88 & $13 \cdot 6$ & $94 \cdot 9$ & $7 \cdot 430$ & $35 \cdot 0$ & $24 \cdot 0$ & 1.06 & 0.08 \\
\hline & Ex. & 200 & 550 & 124 & $30 \cdot 6$ & $\begin{array}{l}96 \cdot 7 \\
95 \cdot 0\end{array}$ & $\begin{array}{l}7 \cdot 420 \\
7 \cdot 415\end{array}$ & $\begin{array}{l}33 \cdot 0 \\
35 \cdot 0\end{array}$ & $\begin{array}{l}23 \cdot 0 \\
23 \cdot 0\end{array}$ & $\begin{array}{l}1.23 \\
1.50\end{array}$ & $\begin{array}{l}0.08 \\
0.10\end{array}$ \\
\hline AH 41 & $\mathbf{R}$ & 0 & 310 & 76 & 7.0 & $95 \cdot 8$ & $7 \cdot 495$ & $35 \cdot 0$ & $27 \cdot 5$ & $1 \cdot 10$ & 0.11 \\
\hline & Ex. & 200 & 750 & 100 & $15 \cdot 4$ & $97 \cdot 4$ & $7 \cdot 465$ & 33.0 & $25 \cdot 0$ & $2 \cdot 20$ & $0 \cdot 13$ \\
\hline & & 400 & 1205 & 124 & $22 \cdot 0$ & $\begin{array}{l}96.4 \\
97.5\end{array}$ & $\begin{array}{l}7 \cdot 445 \\
7 \cdot 435\end{array}$ & $\begin{array}{l}36.0 \\
35.5\end{array}$ & $\begin{array}{l}25.0 \\
24.0\end{array}$ & $\begin{array}{l}2 \cdot 46 \\
2 \cdot 46\end{array}$ & $\begin{array}{l}0.16 \\
0.18\end{array}$ \\
\hline & & 600 & 1510 & 144 & 33.0 & $\begin{array}{l}97 \cdot 4 \\
96.0 \\
96 \cdot 0\end{array}$ & $\begin{array}{l}\mathbf{7 \cdot 4 2 5} \\
\mathbf{7 \cdot 3 7 5} \\
\mathbf{7 \cdot 4 0 5}\end{array}$ & $\begin{array}{l}37.0 \\
39.5 \\
31.0\end{array}$ & $\begin{array}{l}24 \cdot 0 \\
22.5 \\
21 \cdot 0\end{array}$ & $\begin{array}{l}3 \cdot 64 \\
3 \cdot 70 \\
5 \cdot 45\end{array}$ & $\begin{array}{l}0.19 \\
0.22 \\
0.22\end{array}$ \\
\hline & Rec. (5') & & - & 88 & 8.9 & - & $7 \cdot 450$ & $30 \cdot 0$ & $22 \cdot 5$ & - & - \\
\hline VH 33 & $\mathbf{R}$ & 0 & 347 & 76 & $6 \cdot 6$ & $98 \cdot 7$ & $7 \cdot 435$ & $37 \cdot 0$ & $24 \cdot 5$ & - & 0.08 \\
\hline & Ex. & 200 & 710 & 126 & $18 \cdot 1$ & $95 \cdot 9$ & $7 \cdot 435$ & 37.0 & $24 \cdot 5$ & - & 0.11 \\
\hline & & 400 & 1055 & 144 & $21 \cdot 0$ & $\begin{array}{l}95 \cdot 9 \\
94.8\end{array}$ & $\begin{array}{l}7 \cdot 430 \\
7 \cdot 425\end{array}$ & $\begin{array}{l}31 \cdot 0 \\
37 \cdot 0\end{array}$ & $\begin{array}{l}22.5 \\
24.0\end{array}$ & 二 & $\begin{array}{l}0.12 \\
0.14\end{array}$ \\
\hline & & 600 & 1390 & 180 & $32 \cdot 0$ & $\begin{array}{l}88 \cdot 4 \\
79 \cdot 6 \\
77 \cdot 3\end{array}$ & $\begin{array}{l}7 \cdot 385 \\
7 \cdot 395 \\
7 \cdot 375\end{array}$ & $\begin{array}{l}35 \cdot 5 \\
33 \cdot 5 \\
32 \cdot 0\end{array}$ & $\begin{array}{l}21.5 \\
21.0 \\
19.5\end{array}$ & 二 & $\begin{array}{l}0.14 \\
0.15 \\
-\end{array}$ \\
\hline & & & & & & & & & & & \\
\hline
\end{tabular}


TABLE III-cantinued

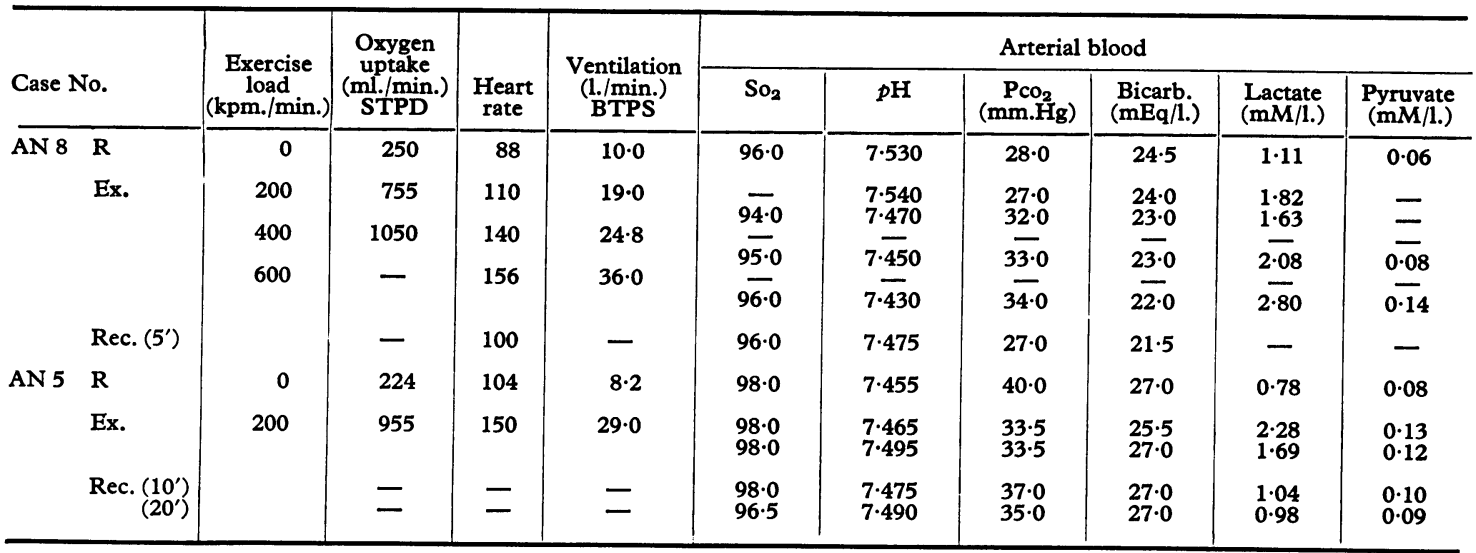

$\mathrm{Kpm} .=$ kilopond metre; Rec. $=$ recovery. Symbols otherwise as in Tables I and II.

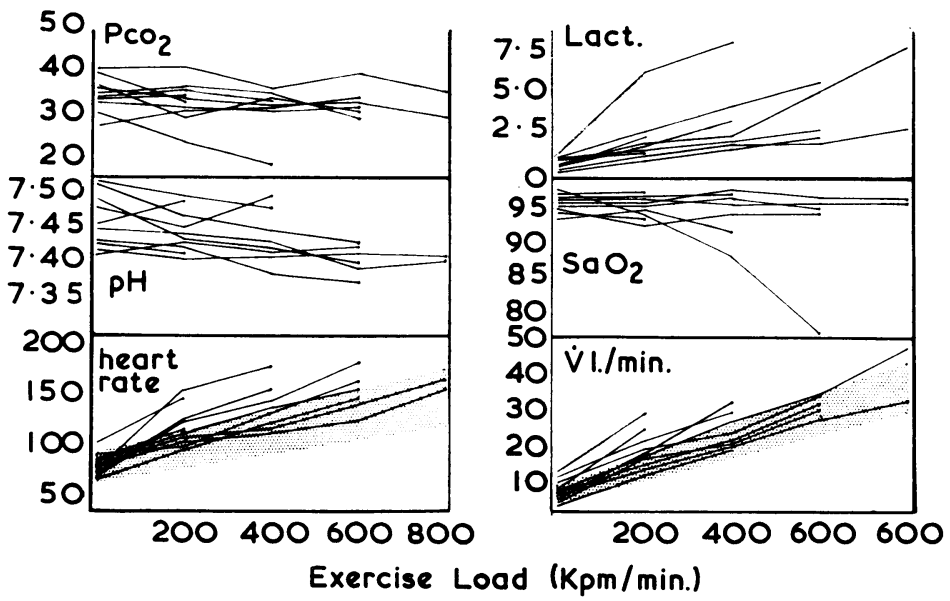

FIG. 9.-Ventilation, heart-rate, and blood chemistry during exercise in the upright position with stepwise increase in load. The normal ranges are shown by the shaded areas (Davies et al., 1966). Excessive ventilation is often seen, and the blood chemistry changes as a result of this.

cases except one (Case VH33) the oxygen saturation remained normal; in the exception the ventilatory response was not unusual. The arterial lactate concentration was excessive in some patients and so were the lactate/pyruvate ratios.

In Fig. 10 we have plotted the lactate/pyruvate ratios and the excess ventilation in all patients whose oxygen uptake was less than $1200 \mathrm{ml} . / \mathrm{min}$. in this study, in both upright and supine positions. We have included for comparison the mean response of patients with pulmonary stenosis (solid line) and mitral stenosis (broken line) in whom similar studies were performed. The patients with left-to-right shunts show a variable response, but most cases behave in a manner similar to that of patients with pulmonary stenosis. The significance of this will be discussed later.

\section{Discussion}

Uncertainty remains in the understanding of the control of ventilation on exercise in normal subjects, as evidenced by the number of recent symposia devoted to the topic. $A$ fortiori the subject is more difficult to unravel in heart disease because of the complexity and interaction of the factors operating. This is particularly the case in patients with left-to-right shunts, in whom pulmonary hypertension, heart failure, lung damage, and impairment of systemic flow may coexist, each being a potential determinant of hyperventilation. 


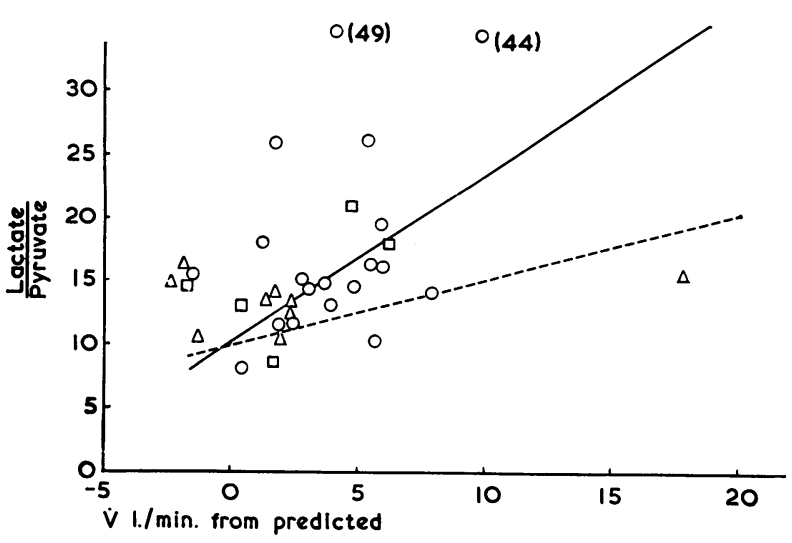

Fig. 10.-Lactate/pyruvate ratio and excess ventilation in all patients with oxygen uptakes below $1200 \mathrm{ml}$./min. The mean response of patients with pulmonary stenosis (solid line) and mitral stenosis (broken line), studied by similar techniques (Gazetopoulos et al., 1966a) is shown for comparison. Symbols and definitions as in Fig. 1 and 2. For discussion see text.

Simultaneous measurement of a number of variables and the comparison of different disease groups may help towards better comprehension, but the problem remains complicated.

Pulmonary plæonæmia of considerable degree may be present in patients with left-to-right shunts without any symptoms whatsoever and with a demonstrably normal ventilatory response to exercise (e.g. Cases AN4, AN6, VN3). Sometimes, however, hyperventilation is observed on effort even in the absence of pulmonary hypertension (i.e. Case AN7). When pulmonary hypertension supervenes in patients with left-to-right shunts, symptoms are usually present, and hyperventilation is frequently, though not invariably, observed on effort (Cases AH44, AH45, PH30). It does not appear from our studies that pulmonary hypertension per se is an important direct causal factor in hyperventilation, and patients are often seen in whom severe pulmonary hypertension is associated with a normal ventilatory response to exercise (Cases AH41, AH43, VH32). A similar conclusion was drawn from similar studies in other forms of heart disease (Gazetopoulos et al., 1966b).

In these studies, therefore, the pattern of ventilatory response to supine exercise has been variable but neither high flow nor pulmonary arterial pressure appear to be powerful independent determinants of hyperventilation. The same variability of response is seen also in upright exercise (Table III).

The influence of pulmonary venous hypertension on the ventilation has been discussed elsewhere
(Gazetopoulos et al., 1966b); increase of left atrial pressure has been found in a minority of patients in this study. In atrial septal defect this is a usual accompaniment of coexisting mitral valve disease or of failure of either ventricle. In ventricular septal defect or patent ductus arteriosus, the presence of mitral stenosis or of left ventricular failure leads to pulmonary venous hypertension. In two of our patients (Cases AH45 and VH34) the increase of left atrial pressure was considerable and both showed marked hyperventilation. Usually, however, the pulmonary venous pressure remains low in patients with septal defects at rest and on effort (Jönsson, Linderholm, and Pinardi, 1957; Stephens, Shafter, and Bliss, 1964) and only exceptionally would the pulmonary venous pressure appear to provide a significant ventilatory stimulus.

Impairment of systemic output has been proposed as a stimulus to ventilation (McIlroy, 1959), but various authors (Cotes, 1955; Arnott, 1963; Sloman and Gandevia, 1964) as well as ourselves (Gazetopoulos et al., 1966b) have failed to find a satisfactorily demonstrable relation between this and the ventilation in different forms of heart disease. The same is true in the present group (Fig. 5). It has been suggested (Gazetopoulos et al., 1966a) that there may be mechanisms of adaptation at tissue level to inadequate oxygen supply, and examination of the consequences of deficient cardiac output, such as the lactate/ pyruvate ratio, could be more reliable indices of tissue hypoxia. A general relationship was found in those studies between this ratio and the ventilation: in isolated low output states, such as pulmonary valve stenosis, higher lactate/pyruvate ratios were seen at comparable degrees of hyperventilation than in patients with pulmonary venous congestion where a more powerful stimulus to ventilation lies in the lung. Most of our patients with left-to-right shunts show a pattern of response in this respect similar to patients with pulmonary stenosis (Fig. 12), suggesting some mechanism connected with tissue hypoxia rather than one related to the lungs in contributing towards hyperventilation in a number of these cases.

The arterial and venous blood gases, as in patients with other types of heart disease discussed elsewhere (Gazetopoulos et al., 1966b), change mainly as a consequence of hyperventilation: the arterial $\mathrm{PcO}_{2}$ may, however, be a fallible index of hyperventilation in these patients owing to coexisting lung disease. Only in severe cyanotic congenital heart disease have we seen variations of blood gases in such a manner as to stimulate ventilation.

We have been impressed with the variability of response to exercise on the part of these patients, 
and have found it difficult to discern which particular factors determine any one individual's response. Of those that are known to encourage hyperventilation, we have seen in this group evidence, in a few cases, of pulmonary venous hypertension and a response similar to that of patients with mitral stenosis. There has been evidence of an impaired cardiac output and resulting tissue hypoxia with a ventilatory response similar to that of patients with pulmonary stenosis. Excessive ventilation not explicable by these manifestations has, however, been observed. We note moreover that dyspnœa has been present without much hyperventilation in some cases.

One major factor which is missing for these considerations is that of organic lung damage and derangement of pulmonary function. This is likely to be a potent factor in symptomatology, and will be discussed in a subsequent paper.

\section{SUMMARY}

Ventilatory, hæmodynamic, and metabolic studies have been performed at rest and during exercise in 34 patients with left-to-right shunts. Most patients showed an excessive ventilatory response to exercise, but this was not apparently related directly to the level of pulmonary arterial pressure or the magnitude of the pulmonary flow. In a minority of patients pulmonary venous hypertension was probably a stimulus to the ventilation.

No particular hæmodynamic parameter or combination of parameters can thus be shown to be directly associated with the manifest hyperventilation of patients with left-to-right shunts, and the cause of this must therefore be sought elsewhere.

The blood gases changed in a manner consequent upon the ventilation. The arterial lactate concentration was excessive in most patients showing hyperventilation, and the possible relation between tissue hypoxia and the ventilatory response has been discussed.

The patients in this study were under the care of Drs. C. G. Baker, R. Kauntze, and D. C. Deuchar, and we thank them for their help. We are particularly grateful to Mrs. J. R. Samuel and the Department of Anæsthetics for the blood-gas analyses, and to the technicians of the Cardiac Department for their assistance.

\section{REFERENCES}

Arnott, W. M. (1963). The lungs in mitral stenosis. Brit. med. $\mathcal{F} ., 2,765$ and 823.

Bruce, R. A., and John, G. G. (1957). Effects of upright posture and exercise on pulmonary hemodynamics in patients with central cardiovascular shunts. Circulation, 16, 776.

Campbell, E. J. M., and Howell, J. B. L. (1963). The sensation of breathlessness. Brit. med. Bull., 19, 36.

Cotes, J. E. (1955). The role of oxygen, carbon dioxide and lactic acid in the ventilatory response to exercise in patients with mitral stenosis. Clin. Sci., 14, 317.

Davies, H., and Gazetopoulos, N. (1965). Dyspncea in cyanotic congenital heart disease. Brit. Heart f., 27, 28.

- , and - (1966). Hæmodynamic changes on exercise in patients with left-to-right shunts. Brit. Heart $\mathcal{F}$. 28, 579.

$\longrightarrow,-$, and Oliver, C. (1965). Ventilation and metabolic response to graduated and prolonged exercise in normal subjects. Clin. Sci., 29, 443.

Donald, K. W., Bishop, J. M., and Wade, O. L. (1954). A study of minute to minute changes of arterio-venous oxygen content difference, oxygen uptake and cardiac output and rate of achievement of a steady state during exercise in rheumatic heart disease. $\mathcal{f}$. clin. Invest., 33, 1146.

Gazetopoulos, N., Davies, H., and Deuchar, D. (1966a). Ventilation in relation to arterial and venous blood chemistry in heart disease. Brit. Heart f., 28, 16.

$\longrightarrow,-$, Oliver, C., and Deuchar, D. (1966b). Ventilation and hæmodynamics in heart disease. Brit. Heart $\mathcal{F}$., 28, 1.

Jonsson, B., Linderholm, H., and Pinardi, G. (1957). Atrial septal defect: A study of physical working capacity and hemodynamics during exercise. Acta med. scand., $159,275$.

McIlroy, M. B. (1959). Dyspnœa and the work of breathing in diseases of the heart and lungs. Progr. cardiovasc. Dis., 1, 284.

New York Heart Association (1953). Nomenclature and Criteria for Diagnosis of Diseases of the Heart and Blood Vessels, 5th ed. New York.

Riley, R. L., Dutton, R. E., Fuleihan, F. J. D., Nath, S., Hurt, H. H., Yoshimoto, C., Sipple, J. H., Permutt, S., and Bromberger-Barnea, B. (1963). Regulation of respiration and blood gases. Ann. N.Y. Acad. Sci., 109, 829.

Sloman, G., and Gandevia, B. (1964). Ventilatory capacity and exercise ventilation in congenital and acquired cardiac disease. Brit. Heart $\mathcal{F}$., 26, 121.

Stephens, N. L., Shafter, H. A., and Bliss, H. A. (1964). Hemodynamic and ventilatory effects of exercise in the upright position in patients with left-to-right shunts. Circulation, 29, 99. 